\title{
IS THE DECOHERENCE OF A SYSTEM THE RESULT OF ITS INTERACTION WITH THE ENVIRONMENT?
}

\author{
MARIO CASTAGNINO, SEBASTIAN FORTIN, AND OLIMPIA LOMBARDI
}

\begin{abstract}
According to a usual reading, decoherence is a process resulting from the interaction between a small system and its large environment where information and energy are dissipated. The particular models treated in the literature on the subject reinforce this idea since, in general, the behavior of a particle immersed in a large "bath" composed by many particles is studied. The aim of this letter is to warn against this usual simplified reading. By means of the analysis of a well-known model, we will show that decoherence may occur in a system interacting with an environment consisting of only one particle.
\end{abstract}

Introduction. The word "decoherence" refers to the quantum process that turns a coherent pure state into a decohered mixed state, which is diagonal in a well defined basis. The phenomenon of decoherence is essential in the account of the emergence of classicality from quantum behavior, since it explains how interference vanishes in an extremely short decoherence time.

The orthodox explanation of the phenomenon is given by the so-called "environment-induced decoherence" (EID) approach ([1], [2], [3], 4]), according to which decoherence is a process resulting from the interaction of a quantum system and its environment. As Zurek states, the environment destroys the coherence between the states of a quantum system by its incessant "monitoring" of the observables associated with the preferred states: it is the environment what "distills" the classical essence from quantum systems (see [3], [4]). In addition, since decoherence only occurs in open quantum systems, it must always be accompanied by other manifestations of openness, such as dissipation of energy and information into the environment.

This way of presenting decoherence has led to a standard reading of the physical meaning of the phenomenon. According to this reading, decoherence is a process resulting from the interaction between a small system and its large environment, where information and energy are dissipated. The particular models treated in the literature on the subject have reinforced this idea since, in general, the behavior of a particle immersed in a large "bath" composed by many particles is studied. The aim of this letter is to warn against this usual simplified reading. By means of the analysis of a well-known model, we will show that decoherence occurs in cases that cannot be described as a small system interacting with a large environment.

Key words and phrases. Quantum decoherence, spin-bath model, relevant observables. 
The spin-bath model. The spin-bath model is a very simple model that has been exactly solved in previous papers (see [1]). We will study it from the general theoretical framework for decoherence presented in a previous work [5]. Let us consider a closed system $U=S+E$ where (i) the system $S$ is a spin-1/2 particle $P$ represented in the Hilbert space $\mathcal{H}_{S}$, and (ii) the environment $E$ is composed of $N$ spin-1/2 particles $P_{i}$, each one of which is represented in its own Hilbert space $\mathcal{H}_{i}$. The complete Hilbert space of the composite system $U$ is, $\mathcal{H}=\mathcal{H}_{S} \bigotimes_{i=1}^{N} \mathcal{H}_{i}$. In the particle $P$, the two eigenstates of the spin operator $S_{S, \vec{v}}$ in direction $\vec{v}$ are $|\uparrow\rangle$ and $|\Downarrow\rangle$, such that $S_{S, \vec{v}}|\Uparrow\rangle=\frac{1}{2}|\Uparrow\rangle$ and $S_{S, \vec{v}}|\Downarrow\rangle=-\frac{1}{2}|\Downarrow\rangle$. In each particle $P_{i}$, the two eigenstates of the corresponding spin operator $S_{i, \vec{v}}$ in direction $\vec{v}$ are $\left|\uparrow_{i}\right\rangle$ and $\left|\downarrow_{i}\right\rangle$, such that $S_{i, \vec{v}}\left|\uparrow_{i}\right\rangle=\frac{1}{2}\left|\uparrow_{i}\right\rangle$ and $S_{i, \vec{v}}\left|\downarrow_{i}\right\rangle=\frac{1}{2}\left|\downarrow_{i}\right\rangle$. Therefore, a pure initial state of $U$ reads

$$
\left|\psi_{0}\right\rangle=(a|\Uparrow\rangle+b|\Downarrow\rangle) \bigotimes_{i=1}^{N}\left(\alpha_{i}\left|\uparrow_{i}\right\rangle+\beta_{i}\left|\downarrow_{i}\right\rangle\right)
$$

where the coefficients $a, b, \alpha_{i}, \beta_{i}$ are such that satisfy $|a|^{2}+|b|^{2}=1$ and $\left|\alpha_{i}\right|^{2}+\left|\beta_{i}\right|^{2}=1$. Usually these numbers (and also the $g_{i}$ below) are taken as aleatory numbers. The self-Hamiltonians $H_{S}$ and $H_{E}$ of $S$ and $E$, respectively, are taken to be zero, then the total Hamiltonian $H=$ $H_{S}+H_{E}+H_{S E}$ of the composite system $U$ results (see [1], [6])

$$
H=H_{S E}=S_{S, \vec{v}} \otimes \sum_{i=1}^{N} 2 g_{i} S_{i, \vec{v}} \bigotimes_{j \neq i}^{N} I_{j}
$$

where $I_{j}$ is the identity operator on the subspace $\mathcal{H}_{j}, S_{S, \vec{v}}=\frac{1}{2}(|\Uparrow\rangle\langle\Uparrow|-| \Downarrow\rangle\langle\Downarrow|)$ and $S_{i, \vec{v}}=$ $\frac{1}{2}\left(\left|\uparrow_{i}\right\rangle\left\langle\uparrow_{i}|-| \downarrow_{i}\right\rangle\left\langle\downarrow_{i}\right|\right)$. Under the action of $H=H_{S E}$, the state $\left|\psi_{0}\right\rangle$ evolves as $|\psi(t)\rangle=$ $a|\Uparrow\rangle\left|\mathcal{E}_{\Uparrow}(t)\right\rangle+b|\Downarrow\rangle\left|\mathcal{E}_{\Downarrow}(t)\right\rangle$ where $\left|\mathcal{E}_{\Uparrow}(t)\right\rangle=\left|\mathcal{E}_{\Downarrow}(-t)\right\rangle$ and

$$
\left|\mathcal{E}_{\Uparrow}(t)\right\rangle=\bigotimes_{i=1}^{N}\left(\alpha_{i} e^{i g_{i} t / 2}\left|\uparrow_{i}\right\rangle+\beta_{i} e^{-i g_{i} t / 2}\left|\downarrow_{i}\right\rangle\right)
$$

If $\mathcal{O}$ is the space of observables of the whole system $U$, let us consider a space of relevant observables $\mathcal{O}_{R} \subset \mathcal{O}$ such that $O_{R} \in \mathcal{O}_{R}$ reads

$$
O_{R}=\left(\begin{array}{c}
s_{\Uparrow \Uparrow}|\Uparrow\rangle\langle\Uparrow| \\
+s_{\Uparrow \Downarrow}|\Uparrow\rangle\langle\Downarrow| \\
+s_{\Downarrow \Uparrow}|\Downarrow\rangle\langle\Uparrow| \\
+s_{\Downarrow \Downarrow}|\Downarrow\rangle\langle\Downarrow|
\end{array}\right) \bigotimes_{i=1}^{N}\left(\begin{array}{c}
\epsilon_{\uparrow \uparrow}^{(i)}\left|\uparrow_{i}\right\rangle\left\langle\uparrow_{i}\right| \\
+\epsilon_{\downarrow \downarrow}^{(i)}\left|\downarrow_{i}\right\rangle\left\langle\downarrow_{i}\right| \\
+\epsilon_{\downarrow \uparrow}^{(i)}\left|\downarrow_{i}\right\rangle\left\langle\uparrow_{i}\right| \\
+\epsilon_{\uparrow \downarrow}^{(i)}\left|\uparrow_{i}\right\rangle\left\langle\downarrow_{i}\right|
\end{array}\right)
$$

Since the operators $O_{R}$ are Hermitian, the diagonal components $s_{\Uparrow \Uparrow}, s_{\Downarrow \Downarrow}, \epsilon_{\uparrow \uparrow}^{(i)}, \epsilon_{\downarrow \downarrow}^{(i)}$ are real numbers and the off-diagonal components are complex numbers satisfying $s_{\Uparrow \Downarrow}=s_{\Downarrow \uparrow}^{*}, \epsilon_{\uparrow \downarrow}^{(i)}=\epsilon_{\downarrow \uparrow}^{(i) *}$. 
Then, the expectation value of the observable $O$ in the state $|\psi(t)\rangle$ can be computed as

$$
\begin{aligned}
\left\langle O_{R}\right\rangle_{\psi(t)}= & \left(|a|^{2} s_{\Uparrow \Uparrow}+|b|^{2} s_{\Downarrow \Downarrow}\right) \Gamma_{0}(t) \\
& +2 \operatorname{Re}\left[a b^{*} s_{\Downarrow \Uparrow} \Gamma_{1}(t)\right]
\end{aligned}
$$

where (see eqs. (23) and (24) in [6])

$$
\begin{aligned}
& \Gamma_{0}(t)=\prod_{i=1}^{N}\left[\begin{array}{c}
\left|\alpha_{i}\right|^{2} \epsilon_{\uparrow \uparrow}^{(i)}+\alpha_{i}{ }^{*} \beta_{i} \epsilon_{\uparrow \downarrow}^{(i)} e^{-i g_{i} t} \\
+\left|\beta_{i}\right|^{2} \epsilon_{\downarrow \downarrow}^{(i)}+\left(\alpha_{i}{ }^{*} \beta_{i} \epsilon_{\uparrow \downarrow}^{(i)}\right)^{*} e^{i g_{i} t}
\end{array}\right] \\
& \Gamma_{1}(t)=\prod_{i=1}^{N}\left[\begin{array}{c}
\left|\alpha_{i}\right|^{2} \epsilon_{\uparrow \uparrow}^{(i)} e^{i g_{i} t}+\left|\beta_{i}\right|^{2} \epsilon_{\downarrow \downarrow}^{(i)} e^{-i g_{i} t} \\
+\alpha_{i}{ }^{*} \beta_{i} \epsilon_{\uparrow \downarrow}^{(i)}+\left(\alpha_{i}{ }^{*} \beta_{i} \epsilon_{\uparrow \downarrow}^{(i)}\right)^{*}
\end{array}\right]
\end{aligned}
$$

As a generalization of the usual presentations, we will study different ways of splitting the whole closed system $U$ into a relevant part and its environment, by considering different choices for the space $\mathcal{O}_{R}$.

Case 1: A large environment that produces decoherence. In the typical situation studied by the EID approach, the system of interest $S$ is simply the particle $P$. Therefore, the relevant observables $O_{R} \in \mathcal{O}_{R}$ are those corresponding to $P$, and are obtained from eq. (44) by making $\epsilon_{\uparrow \uparrow}^{(i)}=\epsilon_{\downarrow \downarrow}^{(i)}=1, \epsilon_{\uparrow \downarrow}^{(i)}=0$ :

$$
O_{R}=\left(\sum_{s, s^{\prime}=\Uparrow, \Downarrow} s_{s s^{\prime}}|s\rangle\left\langle s^{\prime}\right|\right) \bigotimes_{i=1}^{N} I_{i}=O_{S} \bigotimes_{i=1}^{N} I_{i}
$$

The expectation value of these observables is given by

$$
\left\langle O_{R}\right\rangle_{\psi(t)}=|a|^{2} s_{\Uparrow \Uparrow}+|b|^{2} s_{\Downarrow \Downarrow}+2 \operatorname{Re}\left[a b^{*} s_{\Downarrow \Uparrow} r_{1}(t)\right]
$$

where

$$
r_{1}(t)=\prod_{i=1}^{N}\left[\left|\alpha_{i}\right|^{2} e^{i g_{i} t}+\left|\beta_{i}\right|^{2} e^{-i g_{i} t}\right]
$$

By comparing eq. (9) with eq. (5), we see that in this case $\Gamma_{0}(t)=1$ and $\Gamma_{1}(t)=r_{1}(t)$. Moreover,

$$
\left|r_{1}(t)\right|^{2}=\prod_{i=1}^{N}\left(\left|\alpha_{i}\right|^{4}+\left|\beta_{i}\right|^{4}+2\left|\alpha_{i}\right|^{2}\left|\beta_{i}\right|^{2} \cos 2 g_{i} t\right)
$$


Since $\left|\alpha_{i}\right|^{2}+\left|\beta_{i}\right|^{2}=1$, then

$$
\begin{aligned}
& \max _{t}\left(\left|\alpha_{i}\right|^{4}+\left|\beta_{i}\right|^{4}+2\left|\alpha_{i}\right|^{2}\left|\beta_{i}\right|^{2} \cos 2 g_{i} t\right) \\
& =\left(\left(\left|\alpha_{i}\right|^{2}+\left|\beta_{i}\right|^{2}\right)^{2}\right)=1
\end{aligned}
$$

and

$$
\begin{aligned}
& \min _{t}\left(\left|\alpha_{i}\right|^{4}+\left|\beta_{i}\right|^{4}+2\left|\alpha_{i}\right|^{2}\left|\beta_{i}\right|^{2} \cos \left(2 g_{i} t\right)\right) \\
= & \left(\left(\left|\alpha_{i}\right|^{2}-\left|\beta_{i}\right|^{2}\right)^{2}\right)=\left(2\left|\alpha_{i}\right|^{2}-1\right)^{2}
\end{aligned}
$$

If the coefficients $g_{i}, \alpha_{i}$ and $\beta_{i}$ are aleatory numbers, then $\left(\left|\alpha_{i}\right|^{4}+\left|\beta_{i}\right|^{4}+2\left|\alpha_{i}\right|^{2}\left|\beta_{i}\right|^{2} \cos 2 g_{i} t\right)$ is an aleatory number which, if $t \neq 0$, fluctuates between 1 and $\left(2\left|\alpha_{i}\right|^{2}-1\right)^{2}$. Let us note that, since the $\left|\alpha_{i}\right|^{2}$ and the $\left|\beta_{i}\right|^{2}$ are aleatory numbers in the closed interval [0,1], when the environment has many particles (that is, when $N \rightarrow \infty$ ), the statistical value of the cases $\left|\alpha_{i}\right|^{2}=1,\left|\beta_{i}\right|^{2}=1,\left|\alpha_{i}\right|^{2}=0$ and $\left|\beta_{i}\right|^{2}=0$ is zero. In this case, eq. (111) for $\left|r_{1}(t)\right|^{2}$ is an infinite product of numbers belonging to the open interval $(0,1)$. As a consequence (see [3], [4]),

$$
\lim _{N \rightarrow \infty} r_{1}(t)=0
$$

In order to know the time-behavior of the expectation value of eq. (9), we have to compute the time-behavior of $r_{1}(t)$. If we know that $r_{1}(0)=1$ for $N \rightarrow \infty$, and that $\lim _{N \rightarrow \infty} r_{1}(t)=0$ for any $t \neq 0$, it can be expected that, for $N$ finite, $r_{1}(t)$ will evolve in time from $r_{1}(0)=1$ to a very small value. Moreover, $r_{1}(t)$ is a periodic function because it is a product of periodic functions with periods depending on the coefficients $g_{i}$. Nevertheless, since the $g_{i}$ are aleatory, the periods of the individual functions are different and, as a consequence, the recurrence time of $r_{1}(t)$ will be very large, and strongly increasing with the number $N$ of particles.

The time-behavior of $r_{1}(t)$ was computed by means of a numerical simulation, where the aleatory numbers $\left|\alpha_{i}\right|^{2},\left|\beta_{i}\right|^{2}$ and $g_{i}$ were obtained from a generator of aleatory numbers: these generator fixed the value of $\left|\alpha_{i}\right|^{2}$, and the $\left|\beta_{i}\right|^{2}$ were computed as $\left|\beta_{i}\right|^{2}=1-\left|\alpha_{i}\right|^{2}$. The function $r_{1}(t)$ for $N=200$ is plotted in Figure ??, which shows that the particle $P$ decoheres in interaction with an environment of $N$ particles $P_{i}$. This result (see also numerical simulations in 6]) agrees with the standard reading of the phenomenon of decoherence: a single particle in interaction with a large environment of many particles decoheres due precisely to that interaction. 


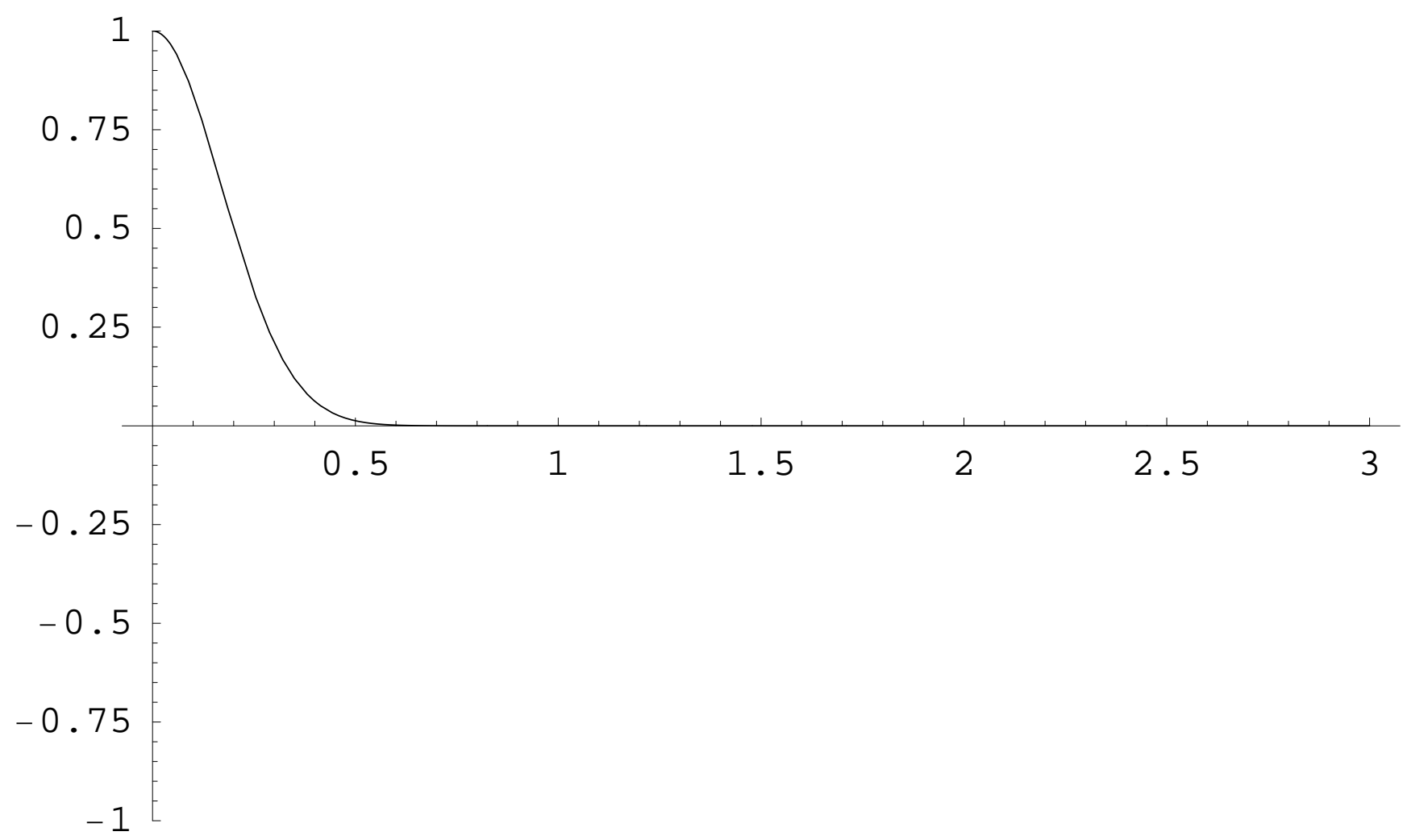

Figure 1. Plot of $\left|r_{1}(t)\right|^{2}$ given by eq. (11), for $N=200$.

Case 2: A large environment with no decoherence. Although in the usual presentations of the model the system of interest is $P$, as in the previous section, we can conceive different ways of splitting the whole system $U$ into a system of interest and an environment. For instance, it may be the case that the measuring arrangement "observes" a particular particle $P_{j}$ of what was previously considered the environment. In this case, the system of interest $S$ is the particle $P_{j}$, and the environment is composed by all the remaining particles, $E=P+\sum_{i \neq j} P_{i}$. Then, the relevant observables $O_{R_{j}} \in \mathcal{O}_{R_{j}} \subset \mathcal{O}$ are only those corresponding to $P_{j}: O_{R_{j}}=I_{S} \otimes O_{S_{j}} \bigotimes_{i \neq j} I_{i}$ where

$$
\begin{aligned}
O_{S_{j}}= & \epsilon_{\uparrow \uparrow}^{(j)}\left|\uparrow_{j}\right\rangle\left\langle\uparrow_{j}\left|+\epsilon_{\downarrow \downarrow}^{(j)}\right| \downarrow_{j}\right\rangle\left\langle\downarrow_{j}\right| \\
& +\epsilon_{\downarrow \uparrow}^{(j)}\left|\downarrow_{j}\right\rangle\left\langle\uparrow_{j}\left|+\epsilon_{\uparrow \downarrow}^{(j)}\right| \uparrow_{j}\right\rangle\left\langle\downarrow_{j}\right|
\end{aligned}
$$

where the coefficients $\epsilon_{\uparrow \uparrow}^{(j)}, \epsilon_{\downarrow \downarrow}^{(j)}, \epsilon_{\downarrow \uparrow}^{(j)}$ are now generic. The expectation value of the observables $O_{R_{j}}$ is given by

$$
\begin{aligned}
\left\langle O_{R_{j}}\right\rangle_{\psi(t)}= & \left|\alpha_{j}\right|^{2} \epsilon_{\uparrow \uparrow}^{(j)}+\left|\beta_{j}\right|^{2} \epsilon_{\downarrow \downarrow}^{(j)} \\
& +\operatorname{Re}\left(\alpha_{j} \beta_{j}^{*} \epsilon_{\uparrow \downarrow}^{(j)} e^{i g_{j} t}\right)
\end{aligned}
$$




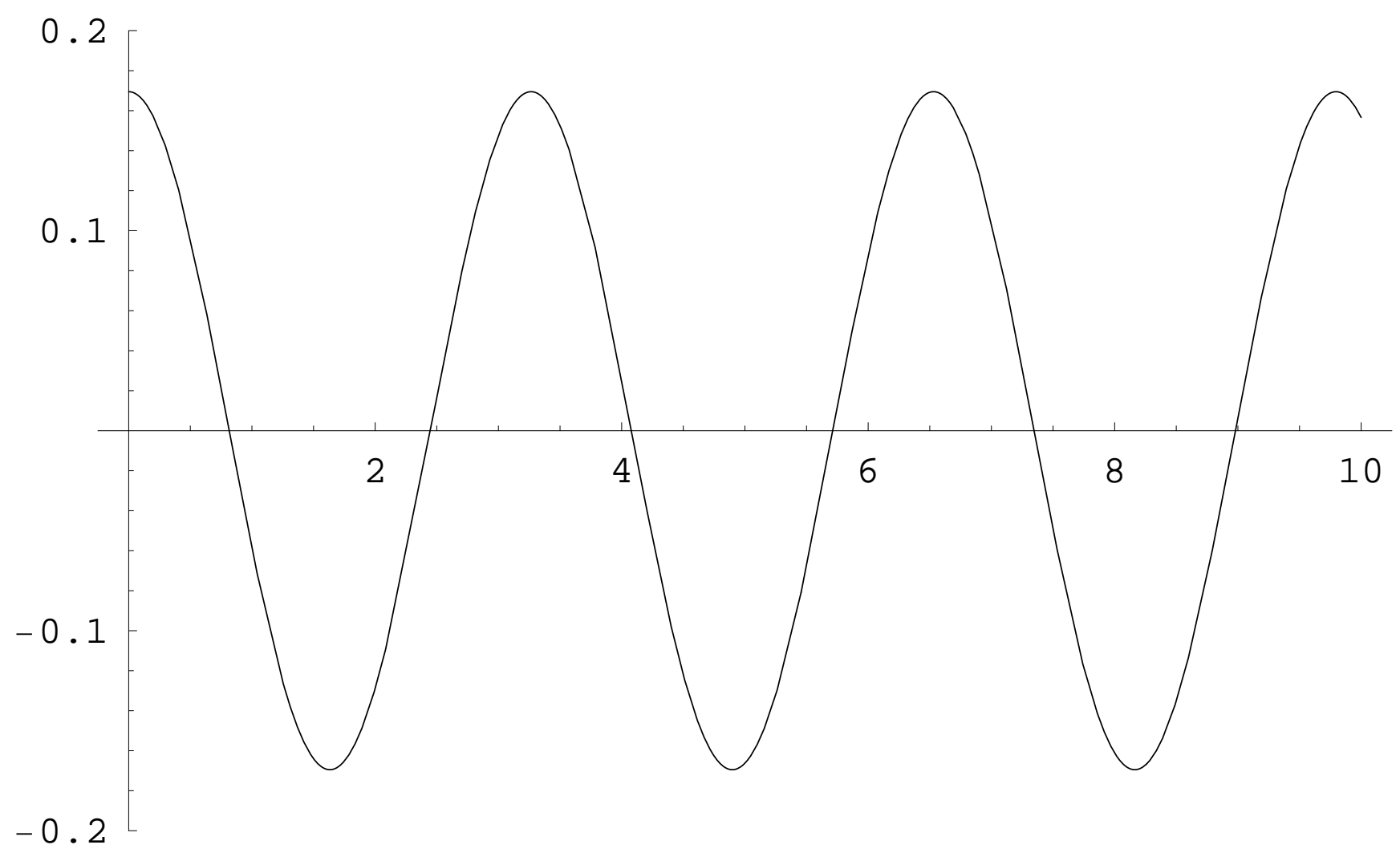

Figure 2. Plot of $r_{2}(t)$ given by eq. (17), for $N \geq 1$.

In order to know the time-evolution of the expectation value of the $O_{R_{j}}$, we have to compute the time-behavior of the third term of eq. (16):

$$
r_{2}(t)=\operatorname{Re}\left(\alpha_{j} \beta_{j}^{*} \epsilon_{\uparrow \downarrow}^{(j)} e^{i g_{j} t}\right)
$$

Let us note that this equation is independent of $N \geq 1$. In this case, numerical simulations are not required to see that $r_{2}(t)$ is an oscillating function which, as a consequence, has no limit for $t \rightarrow \infty$. Nevertheless, in order to illustrate the non decoherence of the system $S$ we show the time-evolution of $r_{2}(t)$ with $N \geq 1$ in Figure ??. In this case, a single particle $S=P_{j}$ with a large environment $E=P+\sum_{i \neq j} P_{i}$ of $N$ particles does not decohere. Nevertheless, this result can be accommodated under the standard reading of the phenomenon of decoherence by saying that $P_{j}$ strongly interacts only with particle $P$, but does not interact with the rest of the particles $P_{i \neq j}$; therefore, the interaction of $S=P_{j}$ with its environment $E=P+\sum_{i \neq j} P_{i}$ is not strong enough to produce decoherence.

Case 3: A small environment that produces decoherence. In this section we consider a measuring arrangement that "observes" a set of particles of the environment, e.g., the $p$ first particles $P_{j}$. In this case, the system of interest is composed by $p$ particles, $S=\sum_{i=1}^{p} P_{i}$, and 
the environment is composed by all the remaining particles, $E=P+\sum_{i=p+1}^{N} P_{i}$. So, in eq. (44), $s_{\Uparrow \Uparrow}=s_{\Downarrow \Downarrow}=1, s_{\Uparrow \Downarrow}=s_{\Downarrow \uparrow}=0$, the coefficients $\epsilon_{\uparrow \uparrow}^{(j)}, \epsilon_{\downarrow \downarrow}^{(j)}, \epsilon_{\downarrow \uparrow}^{(j)}$ are generic for $j \in\{1 \ldots p\}$, and $\epsilon_{\uparrow \uparrow}^{(i)}=\epsilon_{\downarrow \downarrow}^{(i)}=1, \epsilon_{\downarrow \uparrow}^{(i)}=\epsilon_{\uparrow \downarrow}^{(i)}=0$ for $i \in\{p+1 \ldots N\}$. Then, the relevant observables $O_{R} \in \mathcal{O}_{R} \subset \mathcal{O}$ read

$$
O_{R}=I_{S} \otimes\left(\bigotimes_{j=1}^{p} O_{S_{j}}\right) \otimes\left(\bigotimes_{i=p+1}^{N} I_{i}\right)
$$

where $O_{S_{j}}$ is given by eq. (15). Therefore, the expectation value of the relevant observables $O_{R}$ is

$$
\left\langle O_{R}\right\rangle_{\psi(t)}=\prod_{i=1}^{p}\left[\begin{array}{c}
\left|\alpha_{i}\right|^{2} \epsilon_{\uparrow \uparrow}^{(i)}+\alpha_{i}^{*} \beta_{i} \epsilon_{\uparrow \downarrow}^{(i)} e^{-i g_{i} t} \\
+\left|\beta_{i}\right|^{2} \epsilon_{\downarrow \downarrow}^{(i)}+\left(\alpha_{i}^{*} \beta_{i} \epsilon_{\uparrow \downarrow}^{(i)}\right)^{*} e^{i g_{i} t}
\end{array}\right]
$$

Although eq. (19) is very similar to eq. (7), we will compute the time-behavior of that expectation value by means of numerical simulations. In order to simplify the computation, we will consider the particular case where the relevant observables are

$$
O_{R}=I_{S} \otimes\left(\bigotimes_{j=1}^{p} S_{x}^{(j)}\right) \otimes\left(\bigotimes_{i=p+1}^{N} I_{i}\right)
$$

where $S_{x}^{(j)}$ is the projection of the spin onto the $x$-axis of the particle $P_{j}$. Then, $\epsilon_{\uparrow \uparrow}^{(j)}=\epsilon_{\downarrow \downarrow}^{(j)}=0$, and the expectation value reads

$$
\left\langle O_{R}\right\rangle_{\psi(t)}=r_{3}(t)=\prod_{i=1}^{p}\left[2 * \operatorname{Re}\left(\alpha_{i}^{*} \beta_{i} \epsilon_{\uparrow \downarrow}^{(i)} e^{-i g_{i} t}\right)\right]
$$

As in eq. (17), in this equation we can select any $N \geq P$. As in Case 1 (see eq. (10)), in this case the time-dependence of $r_{3}(t)$ is given by a periodic function, whose recurrence time strongly increases with the number of the involved particles.

The time-behavior of $r_{3}(t)$, with $p=4$, is plotted in Figure ??, where we can see a fast decaying followed by fluctuations around zero. As expected, such fluctuations strongly damp off with the increase of the number $p$ of particles, as shown in Figure ?? $(p=8)$ and Figure $5(p=10)$; with $p=200$ the plot turns out to be indistinguishable of that obtained for the decoherence of Case 1 with $N=200$.

The surprising consequence of these results is that the time-behavior is independent of the number $N$ of the particles $P_{i}$, but only depends on the number $p$ of the particles that constitute the system of interest (see eq. (19)). Therefore, we can consider a limit case of $N=p=10$, where the system $S$ is composed by the $p=N=10$ particles and the environment $E$ is a 


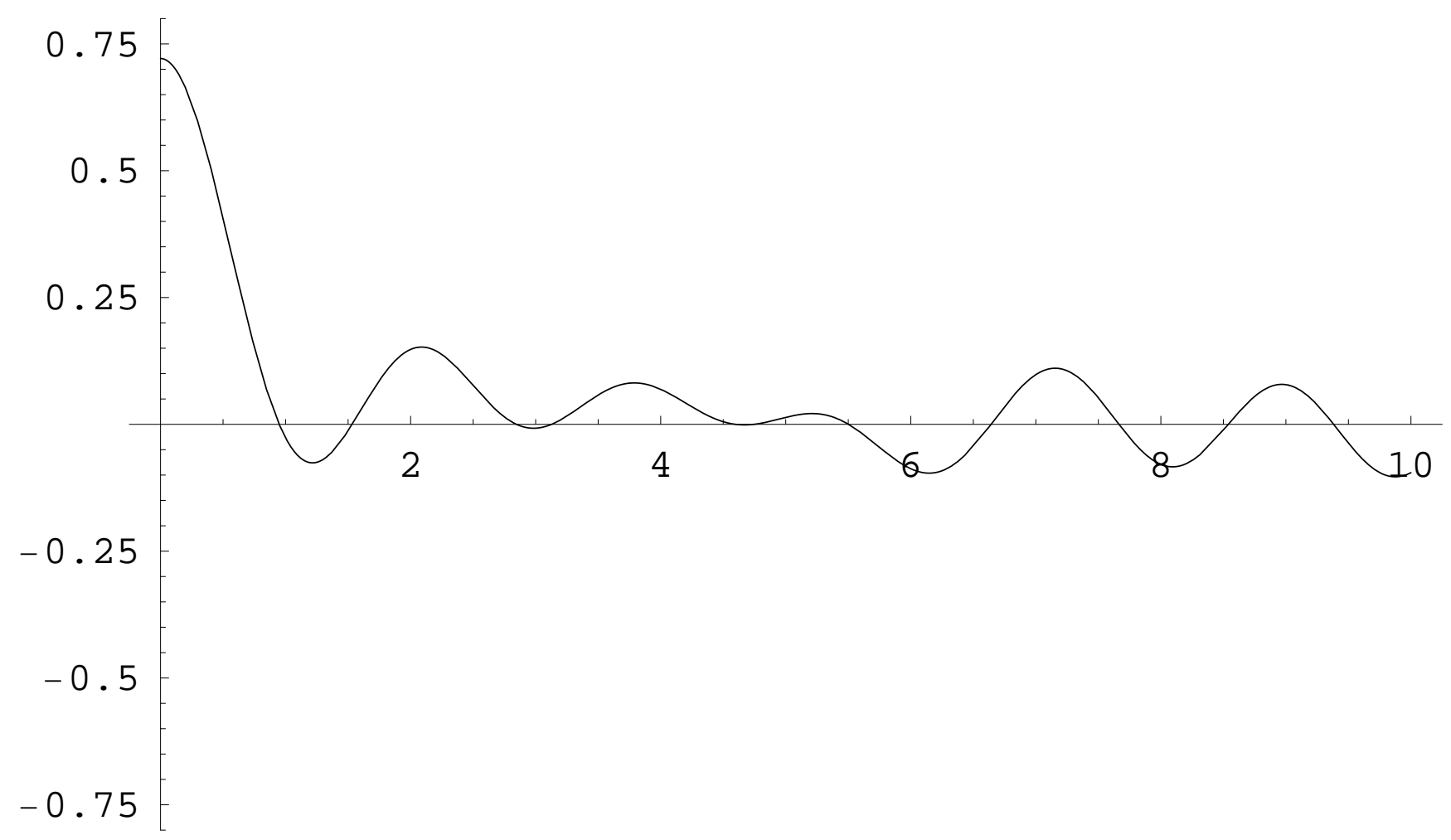

Figure 3. Plot of $r_{3}(t)$ given by eq. (21), for $p=4$.

single particle, $E=P$ : in this case, as shown in Figure ??, we have to say that a system of 10 particles decoheres as the result of its interaction with a single-particle environment. The situation becomes even more striking as the number $p$ increases: with $N=p=200$, the system of 200 particles strongly decoheres in interaction with a single-particle environment. These results can hardly be accommodated under the standard reading of the phenomenon of decoherence, according to which decoherence is produced by the interaction between a small system and a large environment. In other words, this result is in complete contradiction with the usual intuition behind EID.

Conclusions. As some authors point out, the theory of decoherence has became the "new orthodoxy" in the quantum physicists community (see [7]). At present, decoherence is studied and tested in many areas such as atomic physics, quantum optics and condensed matter, and it has acquired a great relevance in quantum computation. This impressive success has led to forget the questions about the physical meaning of decoherence. In general, decoherence is expected to occur only when a small system interacts with a large environment: the dissipation of information and energy from the system to the large environment is what should cause the destruction of the coherence between the states of the system. 


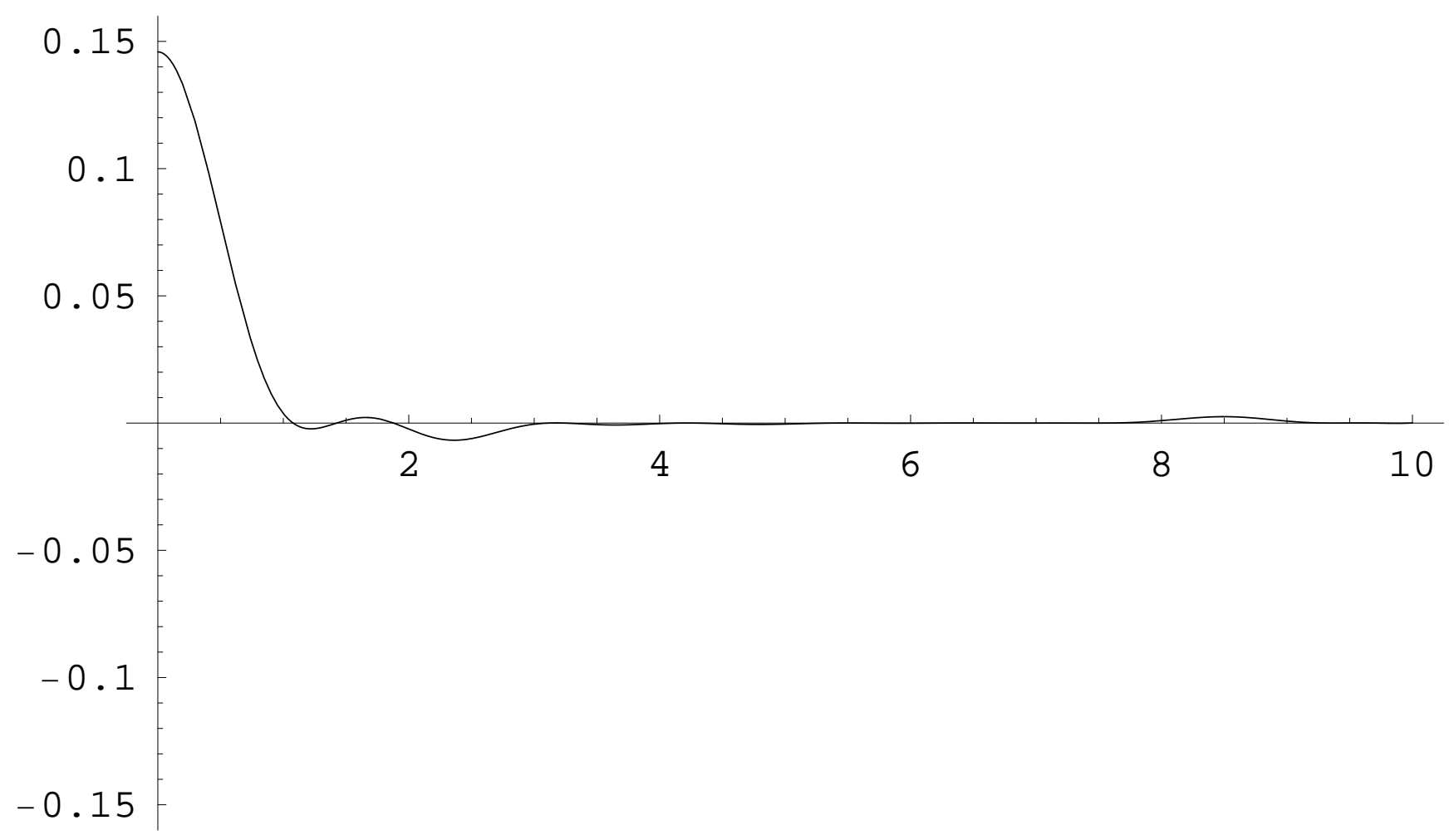

Figure 4. Plot of $r_{3}(t)$ given by eq. (21), for $p=8$.

By studying a well-known model from different perspectives, in this letter we have shown that the usual way of understanding the physical meaning of decoherence is, at least, misguided: a large system in interaction with a small environment may decohere under particular conditions. The general moral of this work is that our understanding of the conceptual foundations of the phenomenon of decoherence is still far from being satisfactory, and the matter deserves to be considered in detail by the physical community.

Acknowledgments. We are very grateful to Roland Omnès and Maximilian Schlösshauer for many comments and criticisms. This research was partially supported by grants of the University of Buenos Aires, CONICET and FONCYT of Argentina.

\section{REFERENCES}

[1] W. H. Zurek, Phys. Rev. D, 26, 1862, 1982.

[2] W. H. Zurek, Progr. Theor. Phys., 89, 281, 1993.

[3] J. P. Paz and W. H. Zurek, "Environment-induced decoherence and the transition from quantum to classical", in Dieter Heiss (ed.), Lecture Notes in Physics, Vol. 587, Heidelberg-Berlin: Springer, 2002.

[4] W. H. Zurek, Rev. Mod. Phys., 75, 715, 2003.

[5] M. Castagnino, S. Fortin, R. Laura and O. Lombardi, Classical and Quantum Gravity, 25, 154002, 2008. 


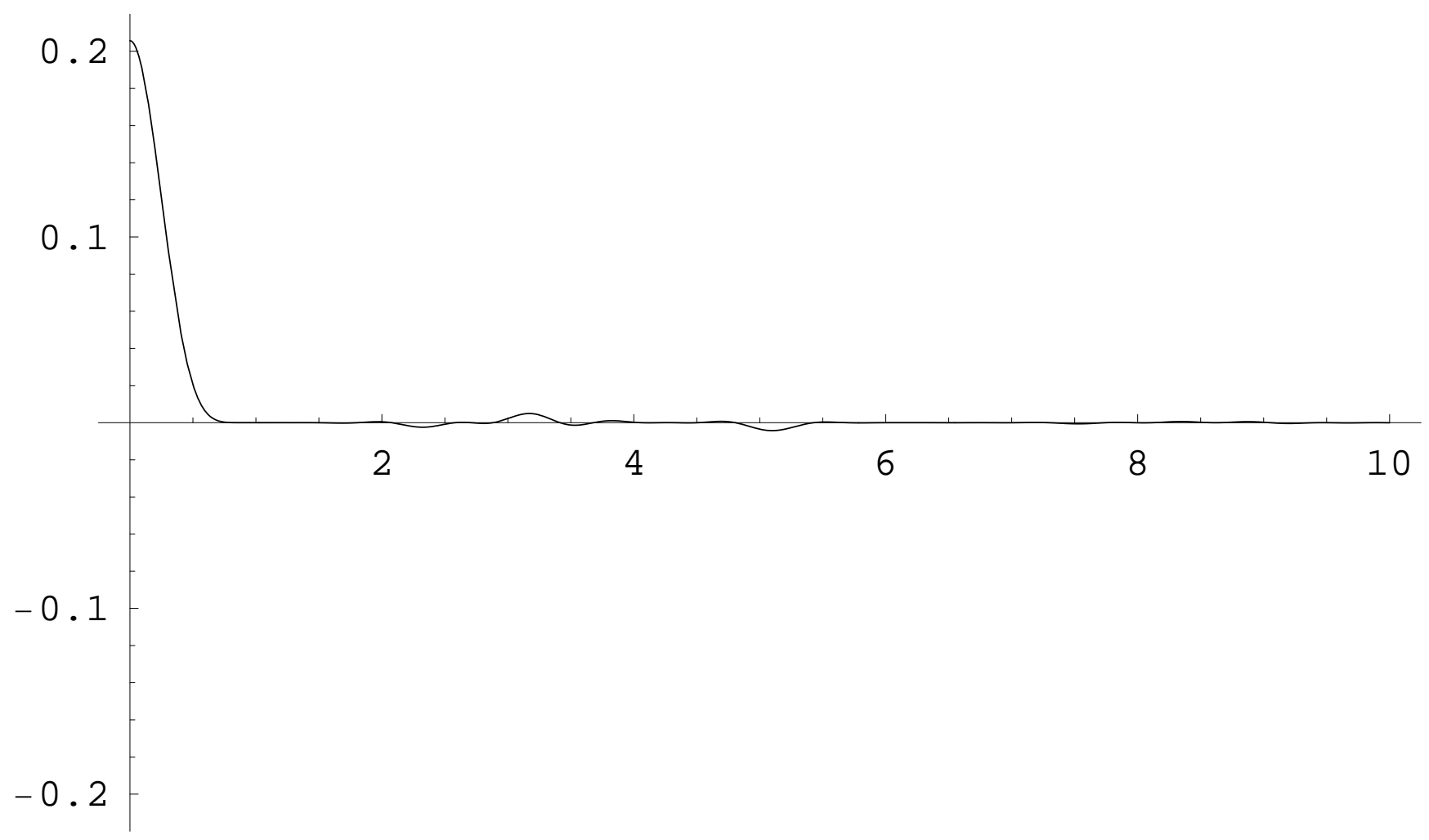

Figure 5. Plot of $r_{3}(t)$ given by eq. (21), for $p=10$.

[6] M. Schlösshauer, Phys. Rev. A, 72, 012109, 2005.

[7] J. Bub, Interpreting the Quantum World, Cambridge: Cambridge University Press, 1997.

CONICET-IAFE-IFIR-Universidad DE Buenos Aires.

COniCET-IAFE-Universidad de Buenos Aires. Email: Sfortin@GmX.net

COniCET-Universidad de Buenos Aires. 